\title{
Barnes' multiple Frobenius-Euler and poly-Bernoulli mixed-type polynomials
}

\section{Dae San Kim ${ }^{1}$, Taekyun Kim²* , Jong-Jin Seo ${ }^{3}$ and Takao Komatsu ${ }^{4}$}

"Correspondence: tkkim@kw.ac.kr 2Department of Mathematics, Kwangwoon University, Seoul, 139-701, Republic of Korea Full list of author information is available at the end of the article

\section{Abstract}

In this paper, we consider Barnes' multiple Frobenius-Euler and poly-Bernoulli mixed-type polynomials. From the properties of Sheffer sequences of these polynomials arising from umbral calculus, we derive new and interesting identities.

MSC: 05A15;05A40; 11B68; 11B75; 65Q05

\section{Introduction}

In this paper, we consider the polynomials $T_{n}^{(r, k)}\left(x \mid a_{1}, \ldots, a_{r} ; \lambda_{1}, \ldots, \lambda_{r}\right)$ whose generating function is given by

$$
\prod_{j=1}^{r}\left(\frac{1-\lambda_{j}}{e^{a_{j} t}-\lambda_{j}}\right) \frac{\operatorname{Li}_{k}\left(1-e^{-t}\right)}{1-e^{-t}} e^{x t}=\sum_{n=0}^{\infty} T_{n}^{(r, k)}\left(x \mid a_{1}, \ldots, a_{r} ; \lambda_{1}, \ldots, \lambda_{r}\right) \frac{t^{n}}{n !}
$$

where $r \in \mathbb{Z}_{>}, k \in \mathbb{Z}, a_{1}, \ldots, a_{r} \neq 0, \lambda_{1}, \ldots, \lambda_{r} \neq 1$ and

$$
\operatorname{Li}_{k}(x)=\sum_{m=1}^{\infty} \frac{x^{m}}{m^{k}}
$$

is the $k$ th polylogarithm function. $T_{n}^{(r, k)}\left(x \mid a_{1}, \ldots, a_{r} ; \lambda_{1}, \ldots, \lambda_{r}\right)$ will be called Barnes' multiple Frobenius-Euler and poly-Bernoulli mixed-type polynomials. When $x=0$, $T_{n}^{(r, k)}\left(a_{1}, \ldots, a_{r} ; \lambda_{1}, \ldots, \lambda_{r}\right)=T_{n}^{(r, k)}\left(0 \mid a_{1}, \ldots, a_{r} ; \lambda_{1}, \ldots, \lambda_{r}\right)$ will be called Barnes' multiple Frobenius-Euler and poly-Bernoulli mixed-type numbers.

Recall that, for every integer $k$, the poly-Bernoulli polynomials $B_{n}^{(k)}(x)$ are defined by the generating function as follows:

$$
\frac{\mathrm{Li}_{k}\left(1-e^{-t}\right)}{1-e^{-t}} e^{x t}=\sum_{n=0}^{\infty} B_{n}^{(k)}(x) \frac{t^{n}}{n !}
$$

([1], $c f$. [2]). Also, as a natural generalization of higher-order Frobenius-Euler polynomials, Barnes' multiple Frobenius-Euler polynomials $H_{n}^{(r)}\left(x \mid a_{1}, \ldots, a_{r} ; \lambda_{1}, \ldots, \lambda_{r}\right)$ are defined by the generating function as follows:

$$
\prod_{j=1}^{r}\left(\frac{1-\lambda_{j}}{e^{a_{j} t}-\lambda_{j}}\right) e^{x t}=\sum_{n=0}^{\infty} H_{n}^{(r)}\left(x \mid a_{1}, \ldots, a_{r} ; \lambda_{1}, \ldots, \lambda_{r}\right) \frac{t^{n}}{n !},
$$

\section{Springer}

○2014 Kim et al.; licensee Springer. This is an Open Access article distributed under the terms of the Creative Commons Attribution License (http://creativecommons.org/licenses/by/2.0), which permits unrestricted use, distribution, and reproduction in any medium, provided the original work is properly cited. 
where $a_{1}, \ldots, a_{r} \neq 0$. Note that the Frobenius-Euler polynomials of order $r, H_{n}^{(r)}(x \mid \lambda)$ are defined by the generating function

$$
\left(\frac{1-\lambda}{e^{t}-\lambda}\right)^{r} e^{x t}=\sum_{n=0}^{\infty} H_{n}^{(r)}(x \mid \lambda) \frac{t^{n}}{n !}
$$

(see, e.g., [3]).

In this paper, we consider Barnes' multiple Frobenius-Euler and poly-Bernoulli mixedtype polynomials. From the properties of Sheffer sequences of these polynomials arising from umbral calculus, we derive new and interesting identities.

\section{Umbral calculus}

Let $\mathbb{C}$ be the complex number field and let $\mathcal{F}$ be the set of all formal power series in the variable $t$ :

$$
\mathcal{F}=\left\{f(t)=\sum_{k=0}^{\infty} \frac{a_{k}}{k !} t^{k} \mid a_{k} \in \mathbb{C}\right\} .
$$

Let $\mathbb{P}=\mathbb{C}[x]$ and let $\mathbb{P}^{*}$ be the vector space of all linear functionals on $\mathbb{P} .\langle L \mid p(x)\rangle$ is the action of the linear functional $L$ on the polynomial $p(x)$, and we recall that the vector space operations on $\mathbb{P}^{*}$ are defined by $\langle L+M \mid p(x)\rangle=\langle L \mid p(x)\rangle+\langle M \mid p(x)\rangle,\langle c L \mid p(x)\rangle=c\langle L \mid p(x)\rangle$, where $c$ is a complex constant in $\mathbb{C}$. For $f(t) \in \mathcal{F}$, let us define the linear functional on $\mathbb{P}$ by setting

$$
\left\langle f(t) \mid x^{n}\right\rangle=a_{n} \quad(n \geq 0) .
$$

In particular,

$$
\left\langle t^{k} \mid x^{n}\right\rangle=n ! \delta_{n, k} \quad(n, k \geq 0)
$$

where $\delta_{n, k}$ is the Kronecker symbol.

For $f_{L}(t)=\sum_{k=0}^{\infty} \frac{\left\langle L \mid x^{k}\right\rangle}{k !} t^{k}$, we have $\left\langle f_{L}(t) \mid x^{n}\right\rangle=\left\langle L \mid x^{n}\right\rangle$. That is, $L=f_{L}(t)$. The map $L \mapsto f_{L}(t)$ is a vector space isomorphism from $\mathbb{P}^{*}$ onto $\mathcal{F}$. Henceforth, $\mathcal{F}$ denotes both the algebra of formal power series in $t$ and the vector space of all linear functionals on $\mathbb{P}$, and so an element $f(t)$ of $\mathcal{F}$ will be thought of as both a formal power series and a linear functional. We call $\mathcal{F}$ the umbral algebra and the umbral calculus is the study of umbral algebra. The order $O(f(t))$ of a power series $f(t)(\neq 0)$ is the smallest integer $k$ for which the coefficient of $t^{k}$ does not vanish. If $O(f(t))=1$, then $f(t)$ is called a delta series; if $O(f(t))=0$, then $f(t)$ is called an invertible series. For $f(t), g(t) \in \mathcal{F}$ with $O(f(t))=1$ and $O(g(t))=0$, there exists a unique sequence $s_{n}(x)\left(\operatorname{deg} s_{n}(x)=n\right)$ such that $\left\langle g(t) f(t)^{k} \mid s_{n}(x)\right\rangle=n ! \delta_{n, k}$ for $n, k \geq 0$. Such a sequence $s_{n}(x)$ is called the Sheffer sequence for $(g(t), f(t))$ which is denoted by $s_{n}(x) \sim(g(t), f(t))$.

For $f(t), g(t) \in \mathcal{F}$ and $p(x) \in \mathbb{P}$, we have

$$
\langle f(t) g(t) \mid p(x)\rangle=\langle f(t) \mid g(t) p(x)\rangle=\langle g(t) \mid f(t) p(x)\rangle
$$


and

$$
f(t)=\sum_{k=0}^{\infty}\left\langle f(t) \mid x^{k}\right\rangle \frac{t^{k}}{k !}, \quad p(x)=\sum_{k=0}^{\infty}\left\langle t^{k} \mid p(x)\right\rangle \frac{x^{k}}{k !}
$$

[4, Theorem 2.2.5]. Thus, by (8), we get

$$
t^{k} p(x)=p^{(k)}(x)=\frac{d^{k} p(x)}{d x^{k}} \text { and } e^{y t} p(x)=p(x+y)
$$

Sheffer sequences are characterized in the generating function [4, Theorem 2.3.4].

Lemma 1 The sequence $s_{n}(x)$ is Sheffer for $(g(t), f(t))$ if and only if

$$
\frac{1}{g(\bar{f}(t))} e^{y \bar{f}(t)}=\sum_{k=0}^{\infty} \frac{s_{k}(y)}{k !} t^{k} \quad(y \in \mathbb{C}),
$$

where $\bar{f}(t)$ is the compositional inverse of $f(t)$.

For $s_{n}(x) \sim(g(t), f(t))$, we have the following equations [4, Theorem 2.3.7, Theorem 2.3.5, Theorem 2.3.9]:

$$
\begin{aligned}
& f(t) s_{n}(x)=n s_{n-1}(x) \quad(n \geq 0), \\
& s_{n}(x)=\sum_{j=0}^{n} \frac{1}{j !}\left\langle g(\bar{f}(t))^{-1} \bar{f}(t)^{j} \mid x^{n}\right\rangle x^{j}, \\
& s_{n}(x+y)=\sum_{j=0}^{n}\left(\begin{array}{l}
n \\
j
\end{array}\right) s_{j}(x) p_{n-j}(y),
\end{aligned}
$$

where $p_{n}(x)=g(t) s_{n}(x)$.

Assume that $p_{n}(x) \sim(1, f(t))$ and $q_{n}(x) \sim(1, g(t))$. Then the transfer formula [4, Corollary 3.8.2] is given by

$$
q_{n}(x)=x\left(\frac{f(t)}{g(t)}\right)^{n} x^{-1} p_{n}(x) \quad(n \geq 1) .
$$

For $s_{n}(x) \sim(g(t), f(t))$ and $r_{n}(x) \sim(h(t), l(t))$, assume that

$$
s_{n}(x)=\sum_{m=0}^{n} C_{n, m} r_{m}(x) \quad(n \geq 0) .
$$

Then we have $[4, \mathrm{p} .132]$

$$
C_{n, m}=\frac{1}{m !}\left\langle\frac{h(\bar{f}(t))}{g(\bar{f}(t))} l(\bar{f}(t))^{m} \mid x^{n}\right\rangle .
$$




\section{Main results}

We now note that $B_{n}^{(k)}(x), H_{n}^{(r)}\left(x \mid a_{1}, \ldots, a_{r} ; \lambda_{1}, \ldots, \lambda_{r}\right)$ and $T_{n}^{(r, k)}\left(x \mid a_{1}, \ldots, a_{r} ; \lambda_{1}, \ldots, \lambda_{r}\right)$ are the Appell sequences for

$$
\begin{aligned}
& g_{k}(t)=\frac{1-e^{-t}}{\operatorname{Li}_{k}\left(1-e^{-t}\right)}, \quad g_{r}(t)=\prod_{j=1}^{r}\left(\frac{e^{a_{j} t}-\lambda_{j}}{1-\lambda_{j}}\right), \\
& g_{r, k}(t)=\prod_{j=1}^{r}\left(\frac{e^{a_{j} t}-\lambda_{j}}{1-\lambda_{j}}\right) \frac{1-e^{-t}}{\operatorname{Li}_{k}\left(1-e^{-t}\right)} .
\end{aligned}
$$

So,

$$
\begin{aligned}
& B_{n}^{(k)}(x) \sim\left(\frac{1-e^{-t}}{\operatorname{Li}_{k}\left(1-e^{-t}\right)}, t\right), \\
& H_{n}^{(r)}\left(x \mid a_{1}, \ldots, a_{r} ; \lambda_{1}, \ldots, \lambda_{r}\right) \sim\left(\prod_{j=1}^{r}\left(\frac{e^{a_{j} t}-\lambda_{j}}{1-\lambda_{j}}\right), t\right), \\
& T_{n}^{(r, k)}\left(x \mid a_{1}, \ldots, a_{r} ; \lambda_{1}, \ldots, \lambda_{r}\right) \sim\left(\prod_{j=1}^{r}\left(\frac{e^{a_{j} t}-\lambda_{j}}{1-\lambda_{j}}\right) \frac{1-e^{-t}}{\mathrm{Li}_{k}\left(1-e^{-t}\right)}, t\right) .
\end{aligned}
$$

In particular, we have

$$
\begin{aligned}
& t B_{n}^{(k)}(x)=\frac{d}{d x} B_{n}^{(k)}(x)=n B_{n-1}^{(k)}(x), \\
& \begin{aligned}
t H_{n}^{(r)}\left(x \mid a_{1}, \ldots, a_{r} ; \lambda_{1}, \ldots, \lambda_{r}\right) & =\frac{d}{d x} H_{n}^{(r)}\left(x \mid a_{1}, \ldots, a_{r} ; \lambda_{1}, \ldots, \lambda_{r}\right) \\
& =n H_{n-1}^{(r)}\left(x \mid a_{1}, \ldots, a_{r} ; \lambda_{1}, \ldots, \lambda_{r}\right), \\
t T_{n}^{(r, k)}\left(x \mid a_{1}, \ldots, a_{r} ; \lambda_{1}, \ldots, \lambda_{r}\right) & =\frac{d}{d x} T_{n}^{(r, k)}\left(x \mid a_{1}, \ldots, a_{r} ; \lambda_{1}, \ldots, \lambda_{r}\right) \\
& =n T_{n-1}^{(r, k)}\left(x \mid a_{1}, \ldots, a_{r} ; \lambda_{1}, \ldots, \lambda_{r}\right) .
\end{aligned}
\end{aligned}
$$

Notice that

$$
\frac{d}{d x} \operatorname{Li}_{k}(x)=\frac{1}{x} \operatorname{Li}_{k-1}(x)
$$

\subsection{Explicit expressions}

Write $H_{n}^{(r)}\left(a_{1}, \ldots, a_{r} ; \lambda_{1}, \ldots, \lambda_{r}\right):=H_{n}^{(r)}\left(0 \mid a_{1}, \ldots, a_{r} ; \lambda_{1}, \ldots, \lambda_{r}\right)$. Let $(n)_{j}=n(n-1) \cdots(n-j+1)$ $(j \geq 1)$ with $(n)_{0}=1$.

\section{Theorem 1}

$$
\begin{aligned}
& T_{n}^{(r, k)}\left(x \mid a_{1}, \ldots, a_{r} ; \lambda_{1}, \ldots, \lambda_{r}\right) \\
& =\sum_{l=0}^{n}\left(\begin{array}{l}
n \\
l
\end{array}\right) B_{l}^{(k)}(x) H_{n-l}^{(r)}\left(a_{1}, \ldots, a_{r} ; \lambda_{1}, \ldots, \lambda_{r}\right) \\
& =\sum_{l=0}^{n}\left(\begin{array}{l}
n \\
l
\end{array}\right) B_{n-l}^{(k)} H_{l}^{(r)}\left(x \mid a_{1}, \ldots, a_{r} ; \lambda_{1}, \ldots, \lambda_{r}\right)
\end{aligned}
$$




$$
\begin{aligned}
= & \sum_{l=0}^{n} \sum_{m=0}^{n} \sum_{j=0}^{m}(-1)^{j}\left(\begin{array}{c}
m \\
j
\end{array}\right)\left(\begin{array}{l}
n \\
l
\end{array}\right) \frac{1}{(m+1)^{k}} H_{n-l}^{(r)}\left(a_{1}, \ldots, a_{r} ; \lambda_{1}, \ldots, \lambda_{r}\right)(x-j)^{l} \\
= & \sum_{l=0}^{n}\left(\sum_{j=l}^{n} \sum_{m=0}^{n-j}(-1)^{n-m-j}\left(\begin{array}{c}
n \\
j
\end{array}\right)\left(\begin{array}{c}
j \\
l
\end{array}\right)\right. \\
& \left.\times \frac{m !}{(m+1)^{k}} S_{2}(n-j, m) H_{j-l}^{(r)}\left(a_{1}, \ldots, a_{r} ; \lambda_{1}, \ldots, \lambda_{r}\right)\right) x^{l} \\
= & \sum_{j=0}^{n}\left(\begin{array}{c}
n \\
j
\end{array}\right) T_{n-j}^{(r, k)}\left(a_{1}, \ldots, a_{r} ; \lambda_{1}, \ldots, \lambda_{r}\right) x^{j} .
\end{aligned}
$$

Proof By (1), (2) and (3), we have

$$
\begin{aligned}
T_{n}^{(r, k)}\left(y \mid a_{1}, \ldots, a_{r} ; \lambda_{1}, \ldots, \lambda_{r}\right) & =\left\langle\sum_{i=0}^{\infty} T_{i}^{(r, k)}\left(y \mid a_{1}, \ldots, a_{r}, \lambda_{1}, \ldots, \lambda_{r}\right) \frac{t^{i}}{i !} \mid x^{n}\right\rangle \\
& =\left\langle\prod_{j=1}^{r}\left(\frac{1-\lambda_{j}}{e^{a_{j} t}-\lambda_{j}}\right) \frac{\mathrm{Li}_{k}\left(1-e^{-t}\right)}{1-e^{-t}} e^{y t} \mid x^{n}\right\rangle \\
& =\left\langle\prod_{j=1}^{r}\left(\frac{1-\lambda_{j}}{e^{a_{j} t}-\lambda_{j}}\right) \mid \frac{\mathrm{Li}_{k}\left(1-e^{-t}\right)}{1-e^{-t}} e^{y t} x^{n}\right\rangle \\
& =\left\langle\prod_{j=1}^{r}\left(\frac{1-\lambda_{j}}{e^{a_{j} t}-\lambda_{j}}\right) \mid \sum_{l=0}^{\infty} B_{l}^{(k)}(y) \frac{t^{l}}{l !} x^{n}\right\rangle \\
& =\sum_{l=0}^{n}\left(\begin{array}{c}
n \\
l
\end{array}\right) B_{l}^{(k)}(y)\left\langle\prod_{j=1}^{r}\left(\frac{1-\lambda_{j}}{e^{a_{j} t}-\lambda_{j}}\right) \mid x^{n-l}\right\rangle \\
& =\sum_{l=0}^{n}\left(\begin{array}{c}
n \\
l
\end{array}\right) B_{l}^{(k)}(y)\left\langle\sum_{i=0}^{\infty} H_{i}^{(r)}\left(a_{1}, \ldots, a_{r} ; \lambda_{1}, \ldots, \lambda_{r}\right) \frac{t^{i}}{i !} \mid x^{n-l}\right\rangle \\
& =\sum_{l=0}^{n}\left(\begin{array}{c}
n \\
l
\end{array}\right) B_{l}^{(k)}(y) H_{n-l}^{(r)}\left(a_{1}, \ldots, a_{r} ; \lambda_{1}, \ldots, \lambda_{r}\right) .
\end{aligned}
$$

So, we get (20).

We also have

$$
\begin{aligned}
T_{n}^{(r, k)}\left(y \mid a_{1}, \ldots, a_{r} ; \lambda_{1}, \ldots, \lambda_{r}\right) & =\left\langle\sum_{i=0}^{\infty} T_{i}^{(r, k)}\left(y \mid a_{1}, \ldots, a_{r} ; \lambda_{1}, \ldots, \lambda_{r}\right) \frac{t^{i}}{i !} \mid x^{n}\right\rangle \\
& =\left\langle\prod_{j=1}^{r}\left(\frac{1-\lambda_{j}}{e^{a_{j} t}-\lambda_{j}}\right) \frac{\operatorname{Li}_{k}\left(1-e^{-t}\right)}{1-e^{-t}} e^{y t} \mid x^{n}\right\rangle \\
& =\left\langle\frac{\operatorname{Li}_{k}\left(1-e^{-t}\right)}{1-e^{-t}} \mid \prod_{j=1}^{r}\left(\frac{1-\lambda_{j}}{e^{a_{j} t}-\lambda_{j}}\right) e^{y t} x^{n}\right\rangle \\
& =\left\langle\frac{\operatorname{Li}_{k}\left(1-e^{-t}\right)}{1-e^{-t}} \mid \sum_{l=0}^{\infty} H_{l}^{(r)}\left(y \mid a_{1}, \ldots, a_{r} ; \lambda_{1}, \ldots, \lambda_{r}\right) \frac{t^{l}}{l !} x^{n}\right\rangle
\end{aligned}
$$




$$
\begin{aligned}
& =\sum_{l=0}^{n}\left(\begin{array}{l}
n \\
l
\end{array}\right) H_{l}^{(r)}\left(y \mid a_{1}, \ldots, a_{r} ; \lambda_{1}, \ldots, \lambda_{r}\right)\left\langle\frac{\operatorname{Li}_{k}\left(1-e^{-t}\right)}{1-e^{-t}} \mid x^{n-l}\right\rangle \\
& =\sum_{l=0}^{n}\left(\begin{array}{l}
n \\
l
\end{array}\right) H_{l}^{(r)}\left(y \mid a_{1}, \ldots, a_{r} ; \lambda_{1}, \ldots, \lambda_{r}\right)\left\langle\sum_{i=0}^{\infty} B_{i}^{(k)} \frac{t^{i}}{i !} \mid x^{n-l}\right\rangle \\
& =\sum_{l=0}^{n}\left(\begin{array}{l}
n \\
l
\end{array}\right) H_{l}^{(r)}\left(y \mid a_{1}, \ldots, a_{r} ; \lambda_{1}, \ldots, \lambda_{r}\right) B_{n-l}^{(k)} .
\end{aligned}
$$

Thus, we get (21).

In [5] we obtained that

$$
\frac{\mathrm{Li}_{k}\left(1-e^{-t}\right)}{1-e^{-t}} x^{n}=\sum_{m=0}^{n} \frac{1}{(m+1)^{k}} \sum_{j=0}^{m}(-1)^{j}\left(\begin{array}{c}
m \\
j
\end{array}\right)(x-j)^{n} .
$$

So,

$$
\begin{aligned}
T_{n}^{(r, k)}\left(x \mid a_{1}, \ldots, a_{r} ; \lambda_{1}, \ldots, \lambda_{r}\right) \\
=\prod_{j=1}^{r}\left(\frac{1-\lambda_{j}}{e^{a_{j} t}-\lambda_{j}}\right) \frac{\mathrm{Li}_{k}\left(1-e^{-t}\right)}{1-e^{-t}} x^{n} \\
=\sum_{m=0}^{n} \frac{1}{(m+1)^{k}} \sum_{j=0}^{m}(-1)^{j}\left(\begin{array}{c}
m \\
j
\end{array}\right) \prod_{j=1}^{r}\left(\frac{1-\lambda_{j}}{e^{a_{j} t}-\lambda_{j}}\right)(x-j)^{n} \\
=\sum_{m=0}^{n} \frac{1}{(m+1)^{k}} \sum_{j=0}^{m}(-1)^{j}\left(\begin{array}{c}
m \\
j
\end{array}\right) \sum_{l=0}^{n}\left(\begin{array}{c}
n \\
l
\end{array}\right) H_{n-l}^{(r)}\left(a_{1}, \ldots, a_{r} ; \lambda_{1}, \ldots, \lambda_{r}\right)(x-j)^{l} \\
=\sum_{l=0}^{n} \sum_{m=0}^{n} \sum_{j=0}^{m}(-1)^{j}\left(\begin{array}{c}
m \\
j
\end{array}\right)\left(\begin{array}{c}
n \\
l
\end{array}\right) \frac{1}{(m+1)^{k}} H_{n-l}^{(r)}\left(a_{1}, \ldots, a_{r} ; \lambda_{1}, \ldots, \lambda_{r}\right)(x-j)^{l},
\end{aligned}
$$

which is identity (22).

In [5] we obtained that

$$
\frac{\mathrm{Li}_{k}\left(1-e^{-t}\right)}{1-e^{-t}} x^{n}=\sum_{j=0}^{n}\left(\sum_{m=0}^{n-j} \frac{(-1)^{n-m-j}}{(m+1)^{k}}\left(\begin{array}{l}
n \\
j
\end{array}\right) m ! S_{2}(n-j, m)\right) x^{j},
$$

where $S_{2}(l, m)$ are the Stirling numbers of the second kind, defined by

$$
\left(e^{t}-1\right)^{m}=m ! \sum_{l=m}^{\infty} S_{2}(l, m) \frac{t^{l}}{l !}
$$

Thus,

$$
\begin{aligned}
T_{n}^{(r, k)}\left(x \mid a_{1}, \ldots, a_{r} ; \lambda_{1}, \ldots, \lambda_{r}\right) \\
\quad=\sum_{j=0}^{n}\left(\sum_{m=0}^{n-j} \frac{(-1)^{n-m-j}}{(m+1)^{k}}\left(\begin{array}{c}
n \\
j
\end{array}\right) m ! S_{2}(n-j, m)\right) \prod_{j=1}^{r}\left(\frac{1-\lambda_{j}}{e^{a_{j} t}-\lambda_{j}}\right) x^{j}
\end{aligned}
$$




$$
\begin{aligned}
& =\sum_{j=0}^{n}\left(\sum_{m=0}^{n-j} \frac{(-1)^{n-m-j}}{(m+1)^{k}}\left(\begin{array}{l}
n \\
j
\end{array}\right) m ! S_{2}(n-j, m)\right) H_{j}^{(r)}\left(x \mid a_{1}, \ldots, a_{r} ; \lambda_{1}, \ldots, \lambda_{r}\right) \\
& =\sum_{j=0}^{n}\left(\sum_{m=0}^{n-j} \frac{(-1)^{n-m-j}}{(m+1)^{k}}\left(\begin{array}{l}
n \\
j
\end{array}\right) m ! S_{2}(n-j, m)\right) \sum_{l=0}^{j}\left(\begin{array}{l}
j \\
l
\end{array}\right) H_{j-l}^{(r)}\left(a_{1}, \ldots, a_{r} ; \lambda_{1}, \ldots, \lambda_{r}\right) x^{l} \\
& =\sum_{l=0}^{n}\left(\sum_{j=l}^{n} \sum_{m=0}^{n-j}(-1)^{n-m-j}\left(\begin{array}{l}
n \\
j
\end{array}\right)\left(\begin{array}{l}
j \\
l
\end{array}\right) \frac{m !}{(m+1)^{k}} S_{2}(n-j, m) H_{j-l}^{(r)}\left(a_{1}, \ldots, a_{r} ; \lambda_{1}, \ldots, \lambda_{r}\right)\right) x^{l},
\end{aligned}
$$

which is identity (23).

By (11) with (16), we have

$$
\begin{aligned}
\left\langle g(\bar{f}(t))^{-1} \bar{f}(t)^{j} \mid x^{n}\right\rangle & =\left\langle\prod_{j=1}^{r}\left(\frac{1-\lambda_{j}}{e^{a_{j} t}-\lambda_{j}}\right) \frac{\mathrm{Li}_{k}\left(1-e^{-t}\right)}{1-e^{-t}} t^{j} \mid x^{n}\right\rangle \\
& =(n)_{j}\left\langle\prod_{j=1}^{r}\left(\frac{1-\lambda_{j}}{e^{a_{j} t}-\lambda_{j}}\right) \frac{\mathrm{Li}_{k}\left(1-e^{-t}\right)}{1-e^{-t}} \mid x^{n-j}\right\rangle \\
& =(n)_{j}\left\langle\sum_{i=0}^{\infty} T_{i}^{(r, k)}\left(a_{1}, \ldots, a_{r} ; \lambda_{1}, \ldots, \lambda_{r}\right) \frac{t^{i}}{i !} \mid x^{n-j}\right\rangle \\
& =(n)_{j} T_{n-j}^{(r, k)}\left(a_{1}, \ldots, a_{r} ; \lambda_{1}, \ldots, \lambda_{r}\right) .
\end{aligned}
$$

Thus, we get (24).

\subsection{Sheffer identity}

\section{Theorem 2}

$$
T_{n}^{(r, k)}\left(x+y \mid a_{1}, \ldots, a_{r} ; \lambda_{1}, \ldots, \lambda_{r}\right)=\sum_{j=0}^{n}\left(\begin{array}{c}
n \\
j
\end{array}\right) T_{j}^{(r, k)}\left(x \mid a_{1}, \ldots, a_{r} ; \lambda_{1}, \ldots, \lambda_{r}\right) y^{n-j}
$$

Proof By (16) with

$$
\begin{aligned}
p_{n}(x) & =\prod_{j=1}^{r}\left(\frac{e^{a_{j} t}-\lambda_{j}}{1-\lambda_{j}}\right) \frac{1-e^{-t}}{\operatorname{Li}_{k}\left(1-e^{-t}\right)} T_{n}^{(r, k)}\left(x \mid a_{1}, \ldots, a_{r} ; \lambda_{1}, \ldots, \lambda_{r}\right) \\
& =x^{n} \sim(1, t),
\end{aligned}
$$

using (12), we have (25).

\subsection{Recurrence}

\section{Theorem 3}

$$
\begin{aligned}
T_{n+1}^{(r, k)}\left(x \mid a_{1}, \ldots, a_{r} ; \lambda_{1}, \ldots, \lambda_{r}\right)= & x T_{n}^{(r, k)}\left(x \mid a_{1}, \ldots, a_{r} ; \lambda_{1}, \ldots, \lambda_{r}\right) \\
& -\sum_{j=1}^{r} \frac{a_{j}}{1-\lambda_{j}} T_{n}^{(r+1, k)}\left(x+a_{j} \mid a_{1}, \ldots, a_{r}, a_{j} ; \lambda_{1}, \ldots, \lambda_{r}, \lambda_{j}\right) \\
& -\frac{1}{n+1} \sum_{l=0}^{n+1}\left(\begin{array}{c}
n+1 \\
l
\end{array}\right) B_{n+1-l}
\end{aligned}
$$




$$
\begin{aligned}
& \times\left(T_{l}^{(r, k)}\left(x \mid a_{1}, \ldots, a_{r} ; \lambda_{1}, \ldots, \lambda_{r}\right)\right. \\
& \left.-T_{l}^{(r, k-1)}\left(x \mid a_{1}, \ldots, a_{r} ; \lambda_{1}, \ldots, \lambda_{r}\right)\right)
\end{aligned}
$$

where $B_{n}$ is the nth ordinary Bernoulli number.

Proof By applying

$$
s_{n+1}(x)=\left(x-\frac{g^{\prime}(t)}{g(t)}\right) \frac{1}{f^{\prime}(t)} s_{n}(x)
$$

[4, Corollary 3.7.2] with (16), we get

$$
T_{n+1}^{(r, k)}\left(x \mid a_{1}, \ldots, a_{r} ; \lambda_{1}, \ldots, \lambda_{r}\right)=\left(x-\frac{g_{r, k}^{\prime}(t)}{g_{r, k}(t)}\right) T_{n}^{(r, k)}\left(x \mid a_{1}, \ldots, a_{r} ; \lambda_{1}, \ldots, \lambda_{r}\right)
$$

Now,

$$
\begin{aligned}
\frac{g_{r, k}^{\prime}(t)}{g_{r, k}(t)} & =\left(\ln g_{r, k}(t)\right)^{\prime} \\
& =\left(\sum_{j=1}^{r} \ln \left(e^{a_{j} t}-\lambda_{j}\right)-\sum_{j=1}^{r} \ln \left(1-\lambda_{j}\right)+\ln \left(1-e^{-t}\right)-\ln \operatorname{Li}_{k}\left(1-e^{-t}\right)\right)^{\prime} \\
& =\sum_{j=1}^{r} \frac{a_{j} e^{a_{j} t}}{e^{a_{j} t}-\lambda_{j}}+\frac{e^{-t}}{1-e^{-t}}\left(1-\frac{\operatorname{Li}_{k-1}\left(1-e^{-t}\right)}{\operatorname{Li}_{k}\left(1-e^{-t}\right)}\right) \\
& =\sum_{j=1}^{r} \frac{a_{j} e^{a_{j} t}}{e^{a_{j} t}-\lambda_{j}}+\frac{t}{e^{t}-1} \frac{\operatorname{Li}_{k}\left(1-e^{-t}\right)-\mathrm{Li}_{k-1}\left(1-e^{-t}\right)}{t \operatorname{Li}_{k}\left(1-e^{-t}\right)} .
\end{aligned}
$$

Since

$$
T_{n}^{(r, k)}\left(x \mid a_{1}, \ldots, a_{r} ; \lambda_{1}, \ldots, \lambda_{r}\right)=\prod_{i=1}^{r}\left(\frac{1-\lambda_{i}}{e^{a_{i} t}-\lambda_{i}}\right) \frac{\operatorname{Li}_{k}\left(1-e^{-t}\right)}{1-e^{-t}} x^{n}
$$

we have

$$
\begin{aligned}
T_{n+1}^{(r, k)} & \left(x \mid a_{1}, \ldots, a_{r} ; \lambda_{1}, \ldots, \lambda_{r}\right) \\
= & x T_{n}^{(r, k)}\left(x \mid a_{1}, \ldots, a_{r} ; \lambda_{1}, \ldots, \lambda_{r}\right) \\
& -\sum_{j=1}^{r} \frac{a_{j} e^{a_{j} t}}{1-\lambda_{j}} \frac{1-\lambda_{j}}{e^{a_{j} t}-\lambda_{j}} \prod_{i=1}^{r}\left(\frac{1-\lambda_{i}}{e^{a_{i} t}-\lambda_{i}}\right) \frac{\mathrm{Li}_{k}\left(1-e^{-t}\right)}{1-e^{-t}} x^{n} \\
& -\frac{t}{e^{t}-1} \prod_{i=1}^{r}\left(\frac{1-\lambda_{i}}{e^{a_{i} t}-\lambda_{i}}\right) \frac{\mathrm{Li}_{k}\left(1-e^{-t}\right)-\mathrm{Li}_{k-1}\left(1-e^{-t}\right)}{t\left(1-e^{-t}\right)} x^{n} .
\end{aligned}
$$

Since

$$
\frac{\operatorname{Li}_{k}\left(1-e^{-t}\right)-\mathrm{Li}_{k-1}\left(1-e^{-t}\right)}{1-e^{-t}}=\left(\frac{1}{2^{k}}-\frac{1}{2^{k-1}}\right) t+\cdots
$$


is a delta series, we get

$$
\frac{\mathrm{Li}_{k}\left(1-e^{-t}\right)-\mathrm{Li}_{k-1}\left(1-e^{-t}\right)}{t\left(1-e^{-t}\right)} x^{n}=\frac{1}{n+1} \frac{\mathrm{Li}_{k}\left(1-e^{-t}\right)-\mathrm{Li}_{k-1}\left(1-e^{-t}\right)}{1-e^{-t}} x^{n+1} .
$$

Therefore, by

$$
\frac{t}{e^{t}-1} x^{n+1}=B_{n+1}(x)=\sum_{l=0}^{n+1}\left(\begin{array}{c}
n+1 \\
l
\end{array}\right) B_{n+1-l} x^{l}
$$

we obtain

$$
\begin{aligned}
T_{n+1}^{(r, k)}( & \left.x \mid a_{1}, \ldots, a_{r} ; \lambda_{1}, \ldots, \lambda_{r}\right) \\
= & x T_{n}^{(r, k)}\left(x \mid a_{1}, \ldots, a_{r} ; \lambda_{1}, \ldots, \lambda_{r}\right)-\sum_{j=1}^{r} \frac{a_{j}}{1-\lambda_{j}} T_{n}^{(r+1, k)}\left(x+a_{j} \mid a_{1}, \ldots, a_{r}, a_{j} ; \lambda_{1}, \ldots, \lambda_{r}, \lambda_{j}\right) \\
& -\frac{1}{n+1} \prod_{i=1}^{r}\left(\frac{1-\lambda_{i}}{e^{a_{i} t}-\lambda_{i}}\right) \frac{\mathrm{Li}_{k}\left(1-e^{-t}\right)-\mathrm{Li}_{k-1}\left(1-e^{-t}\right)}{1-e^{-t}} \frac{t}{e^{t}-1} x^{n+1} \\
= & x T_{n}^{(r, k)}\left(x \mid a_{1}, \ldots, a_{r} ; \lambda_{1}, \ldots, \lambda_{r}\right)-\sum_{j=1}^{r} \frac{a_{j}}{1-\lambda_{j}} T_{n}^{(r+1, k)}\left(x+a_{j} \mid a_{1}, \ldots, a_{r}, a_{j} ; \lambda_{1}, \ldots, \lambda_{r}, \lambda_{j}\right) \\
& -\frac{1}{n+1} \sum_{l=0}^{n+1}\left(\begin{array}{c}
n+1 \\
l
\end{array}\right) B_{n+1-l} \\
& \times\left(T_{l}^{(r, k)}\left(x \mid a_{1}, \ldots, a_{r} ; \lambda_{1}, \ldots, \lambda_{r}\right)-T_{l}^{(r, k-1)}\left(x \mid a_{1}, \ldots, a_{r} ; \lambda_{1}, \ldots, \lambda_{r}\right)\right),
\end{aligned}
$$

which is identity (26).

\subsection{A more relation}

\section{Theorem 4}

$$
\begin{aligned}
T_{n}^{(r, k)}( & \left.x \mid a_{1}, \ldots, a_{r} ; \lambda_{1}, \ldots, \lambda_{r}\right) \\
= & x T_{n-1}^{(r, k)}\left(x \mid a_{1}, \ldots, a_{r} ; \lambda_{1}, \ldots, \lambda_{r}\right) \\
& -\sum_{j=1}^{r} \frac{a_{j}}{1-\lambda_{j}} T_{n-1}^{(r+1, k)}\left(x+a_{j} \mid a_{1}, \ldots, a_{r}, a_{j} ; \lambda_{1}, \ldots, \lambda_{r}, \lambda_{j}\right) \\
& -\frac{1}{n} \sum_{l=0}^{n}\left(\begin{array}{c}
n \\
l
\end{array}\right) B_{n-l}\left(T_{l}^{(r, k)}\left(x \mid a_{1}, \ldots, a_{r} ; \lambda_{1}, \ldots, \lambda_{r}\right)\right. \\
& \left.-T_{l}^{(r, k-1)}\left(x \mid a_{1}, \ldots, a_{r} ; \lambda_{1}, \ldots, \lambda_{r}\right)\right) .
\end{aligned}
$$

Proof For $n \geq 1$, we have

$$
\begin{aligned}
T_{n}^{(r, k)}\left(y \mid a_{1}, \ldots, a_{r} ; \lambda_{1}, \ldots, \lambda_{r}\right) & =\left\langle\sum_{l=0}^{\infty} T_{l}^{(r, k)}\left(y \mid a_{1}, \ldots, a_{r} ; \lambda_{1}, \ldots, \lambda_{r}\right) \frac{t^{l}}{l !} \mid x^{n}\right\rangle \\
& =\left\langle\prod_{j=1}^{r}\left(\frac{1-\lambda_{j}}{e^{a_{j} t}-\lambda_{j}}\right) \frac{\mathrm{Li}_{k}\left(1-e^{-t}\right)}{1-e^{-t}} e^{y t} \mid x^{n}\right\rangle
\end{aligned}
$$




$$
\begin{aligned}
= & \left\langle\partial_{t}\left(\prod_{j=1}^{r}\left(\frac{1-\lambda_{j}}{e^{a_{j} t}-\lambda_{j}}\right) \frac{\mathrm{Li}_{k}\left(1-e^{-t}\right)}{1-e^{-t}} e^{y t}\right) \mid x^{n-1}\right\rangle \\
= & \left\langle\left(\partial_{t} \prod_{j=1}^{r}\left(\frac{1-\lambda_{j}}{e^{a_{j} t}-\lambda_{j}}\right)\right) \frac{\mathrm{Li}_{k}\left(1-e^{-t}\right)}{1-e^{-t}} e^{y t} \mid x^{n-1}\right\rangle \\
& +\left\langle\prod_{j=1}^{r}\left(\frac{1-\lambda_{j}}{e^{a_{j} t}-\lambda_{j}}\right)\left(\partial_{t} \frac{\operatorname{Li}_{k}\left(1-e^{-t}\right)}{1-e^{-t}}\right) e^{y t} \mid x^{n-1}\right\rangle \\
& +\left\langle\prod_{j=1}^{r}\left(\frac{1-\lambda_{j}}{e^{a_{j} t}-\lambda_{j}}\right) \frac{\mathrm{Li}_{k}\left(1-e^{-t}\right)}{1-e^{-t}\left(\partial_{t} e^{y t}\right)\left|x^{n-1}\right\rangle}\right. \\
= & y T_{n-1}^{(r, k)}\left(y \mid a_{1}, \ldots, a_{r} ; \lambda_{1}, \ldots, \lambda_{r}\right) \\
& +\left\langle\left(\partial_{t} \prod_{j=1}^{r}\left(\frac{1-\lambda_{j}}{e^{a_{j} t}-\lambda_{j}}\right)\right) \frac{\mathrm{Li}_{k}\left(1-e^{-t}\right)}{1-e^{-t}} e^{y t} \mid x^{n-1}\right\rangle \\
& +\left\langle\prod_{j=1}^{r}\left(\frac{1-\lambda_{j}}{e^{a_{j} t}-\lambda_{j}}\right)\left(\partial_{t} \frac{\mathrm{Li}_{k}\left(1-e^{-t}\right)}{1-e^{-t}}\right) e^{y t} \mid x^{n-1}\right\rangle .
\end{aligned}
$$

Observe that

$$
\begin{aligned}
\partial_{t} \prod_{j=1}^{r}\left(\frac{1-\lambda_{j}}{e^{a_{j} t}-\lambda_{j}}\right) & =\prod_{j=1}^{r}\left(1-\lambda_{j}\right) \partial_{t}\left(\frac{1}{\prod_{j=1}^{r}\left(e^{a_{j} t}-\lambda_{j}\right)}\right) \\
& =\prod_{j=1}^{r}\left(1-\lambda_{j}\right) \frac{-\left(\prod_{j=1}^{r}\left(e^{a_{j} t}-\lambda_{j}\right)\right)^{\prime}}{\left(\prod_{j=1}^{r}\left(e^{a_{j} t}-\lambda_{j}\right)\right)^{2}} \\
& =-\prod_{j=1}^{r}\left(1-\lambda_{j}\right) \frac{\sum_{j=1}^{r} a_{j} e^{a_{j} t} \prod_{i \neq j}\left(e^{a_{i} t}-\lambda_{i}\right)}{\left(\prod_{j=1}^{r}\left(e^{a_{j} t}-\lambda_{j}\right)\right)^{2}} \\
& =-\sum_{j=1}^{r} \frac{a_{j} e^{a_{j} t}}{e^{a_{j} t}-\lambda_{j}} \prod_{i=1}^{r}\left(\frac{1-\lambda_{i}}{e^{a_{i} t}-\lambda_{i}}\right) \\
& =-\sum_{j=1}^{r} \frac{a_{j} e^{a_{j} t}}{1-\lambda_{j}} \frac{1-\lambda_{j}}{e^{a_{j} t}-\lambda_{j}} \prod_{i=1}^{r}\left(\frac{1-\lambda_{i}}{e^{a_{i} t}-\lambda_{i}}\right) .
\end{aligned}
$$

Thus,

$$
\begin{aligned}
& \left\langle\left(\partial_{t} \prod_{j=1}^{r}\left(\frac{1-\lambda_{j}}{e^{a_{j} t}-\lambda_{j}}\right)\right) \frac{\mathrm{Li}_{k}\left(1-e^{-t}\right)}{1-e^{-t}} e^{y t} \mid x^{n-1}\right\rangle \\
& \quad=-\sum_{j=1}^{r} \frac{a_{j}}{1-\lambda_{j}}\left\langle\frac{1-\lambda_{j}}{e^{a_{j} t}-\lambda_{j}} \prod_{i=1}^{r}\left(\frac{1-\lambda_{i}}{e^{a_{i} t}-\lambda_{i}}\right) \frac{\mathrm{Li}_{k}\left(1-e^{-t}\right)}{1-e^{-t}} e^{\left(y+a_{j}\right) t} \mid x^{n-1}\right\rangle \\
& \quad=-\sum_{j=1}^{r} \frac{a_{j}}{1-\lambda_{j}} T_{n-1}^{(r+1, k)}\left(y+a_{j} \mid a_{1}, \ldots, a_{r}, a_{j} ; \lambda_{1}, \ldots, \lambda_{r}, \lambda_{j}\right) .
\end{aligned}
$$


Since

$$
\begin{aligned}
\partial_{t}\left(\frac{\operatorname{Li}_{k}\left(1-e^{-t}\right)}{1-e^{-t}}\right) & =\frac{e^{-t}\left(\operatorname{Li}_{k-1}\left(1-e^{-t}\right)-\operatorname{Li}_{k}\left(1-e^{-t}\right)\right)}{\left(1-e^{-t}\right)^{2}} \\
& =\frac{t}{e^{t}-1} \frac{\operatorname{Li}_{k-1}\left(1-e^{-t}\right)-\mathrm{Li}_{k}\left(1-e^{-t}\right)}{t\left(1-e^{-t}\right)}
\end{aligned}
$$

and the fact that

$$
\frac{\mathrm{Li}_{k-1}\left(1-e^{-t}\right)-\mathrm{Li}_{k}\left(1-e^{-t}\right)}{1-e^{-t}}=\left(\frac{1}{2^{k-1}}-\frac{1}{2^{k}}\right) t+\cdots
$$

is a delta series, we have

$$
\begin{aligned}
& \left\langle\prod_{j=1}^{r}\left(\frac{1-\lambda_{j}}{e^{a_{j} t}-\lambda_{j}}\right)\left(\partial_{t} \frac{\mathrm{Li}_{k}\left(1-e^{-t}\right)}{1-e^{-t}}\right) e^{y t} \mid x^{n-1}\right\rangle \\
& \quad=\left\langle\prod_{j=1}^{r}\left(\frac{1-\lambda_{j}}{e^{a_{j} t}-\lambda_{j}}\right) \frac{t}{e^{t}-1} \frac{\mathrm{Li}_{k-1}\left(1-e^{-t}\right)-\mathrm{Li}_{k}\left(1-e^{-t}\right)}{t\left(1-e^{-t}\right)} e^{y t} \mid x^{n-1}\right\rangle \\
& \quad=\left\langle\prod_{j=1}^{r}\left(\frac{1-\lambda_{j}}{e^{a_{j} t}-\lambda_{j}}\right) \frac{t}{e^{t}-1} \frac{\mathrm{Li}_{k-1}\left(1-e^{-t}\right)-\mathrm{Li}_{k}\left(1-e^{-t}\right.}{1-e^{-t}} e^{y t} \mid \frac{x^{n}}{n}\right\rangle \\
& \quad=\frac{1}{n}\left\langle\prod_{j=1}^{r}\left(\frac{1-\lambda_{j}}{e^{a_{j} t}-\lambda_{j}}\right) \frac{\mathrm{Li}_{k-1}\left(1-e^{-t}\right)-\mathrm{Li}_{k}\left(1-e^{-t}\right)}{1-e^{-t}} e^{y t} \mid \frac{t}{e^{t}-1} x^{n}\right\rangle \\
& \quad=\frac{1}{n} \sum_{l=0}^{n}\left(\begin{array}{l}
n \\
l
\end{array}\right) B_{n-l}\left\langle\prod_{j=1}^{r}\left(\frac{1-\lambda_{j}}{e^{a_{j} t}-\lambda_{j}}\right) \frac{\mathrm{Li}_{k-1}\left(1-e^{-t}\right)-\mathrm{Li}_{k}\left(1-e^{-t}\right)}{1-e^{-t}} e^{y t} \mid x^{l}\right\rangle \\
& \quad=\frac{1}{n} \sum_{l=0}^{n}\left(\begin{array}{l}
n \\
l
\end{array}\right) B_{n-l}\left(T_{l}^{(r, k-1)}\left(y \mid a_{1}, \ldots, a_{r} ; \lambda_{1}, \ldots, \lambda_{r}\right)-T_{l}^{(r, k)}\left(y \mid a_{1}, \ldots, a_{r} ; \lambda_{1}, \ldots, \lambda_{r}\right)\right) .
\end{aligned}
$$

Therefore, we obtain the desired result.

Remark After $n$ is replaced by $n+1$, identity (27) becomes the recurrence formula (26).

\subsection{Relations with poly-Bernoulli numbers and Barnes' multiple Bernoulli numbers}

\section{Theorem 5}

$$
\begin{aligned}
& \sum_{m=0}^{n}(-1)^{n-m}\left(\begin{array}{c}
n+1 \\
m
\end{array}\right) T_{m}^{(r, k)}\left(a_{1}, \ldots, a_{r} ; \lambda_{1}, \ldots, \lambda_{r}\right) \\
& \quad=\sum_{l=0}^{n} \sum_{m=0}^{l}(-1)^{l-m}\left(\begin{array}{c}
l \\
m
\end{array}\right)\left(\begin{array}{c}
n+1 \\
l+1
\end{array}\right) B_{m}^{(k-1)} H_{n-l}^{(r)}\left(a_{1}, \ldots, a_{r} ; \lambda_{1}, \ldots, \lambda_{r}\right) .
\end{aligned}
$$

Proof We shall compute

$$
\left\langle\prod_{j=1}^{r}\left(\frac{1-\lambda_{j}}{e^{a_{j} t}-\lambda_{j}}\right) \operatorname{Li}_{k}\left(1-e^{-t}\right) \mid x^{n+1}\right\rangle
$$


in two different ways. On the one hand,

$$
\begin{aligned}
& \left\langle\prod_{j=1}^{r}\left(\frac{1-\lambda_{j}}{e^{a_{j} t}-\lambda_{j}}\right) \operatorname{Li}_{k}\left(1-e^{-t}\right) \mid x^{n+1}\right\rangle \\
& \quad=\left\langle\prod_{j=1}^{r}\left(\frac{1-\lambda_{j}}{e^{a_{j} t}-\lambda_{j}}\right) \frac{\operatorname{Li}_{k}\left(1-e^{-t}\right)}{1-e^{-t}} \mid\left(1-e^{-t}\right) x^{n+1}\right\rangle \\
& \quad=\left\langle\prod_{j=1}^{r}\left(\frac{1-\lambda_{j}}{e^{a_{j} t}-\lambda_{j}}\right) \frac{\operatorname{Li}_{k}\left(1-e^{-t}\right)}{1-e^{-t}} \mid x^{n+1}-(x-1)^{n+1}\right\rangle \\
& \quad=\sum_{m=0}^{n}\left(\begin{array}{c}
n+1 \\
m
\end{array}\right)(-1)^{n-m}\left\langle\prod_{j=1}^{r}\left(\frac{1-\lambda_{j}}{e^{a_{j} t}-\lambda_{j}}\right) \frac{\mathrm{Li}_{k}\left(1-e^{-t}\right)}{1-e^{-t}} \mid x^{m}\right\rangle \\
& \quad=\sum_{m=0}^{n}\left(\begin{array}{c}
n+1 \\
m
\end{array}\right)(-1)^{n-m} T_{m}^{(r, k)}\left(a_{1}, \ldots, a_{r} ; \lambda_{1}, \ldots, \lambda_{r}\right) .
\end{aligned}
$$

On the other hand,

$$
\begin{aligned}
\left\langle\prod_{j=1}^{r}\left(\frac{1-\lambda_{j}}{e^{a_{j} t}-\lambda_{j}}\right) \mathrm{Li}_{k}\left(1-e^{-t}\right) \mid x^{n+1}\right\rangle \\
\quad=\left\langle\mathrm{Li}_{k}\left(1-e^{-t}\right) \mid \prod_{j=1}^{r}\left(\frac{1-\lambda_{j}}{e^{a_{j} t}-\lambda_{j}}\right) x^{n+1}\right\rangle \\
=\left\langle\mathrm{Li}_{k}\left(1-e^{-t}\right) \mid H_{n+1}^{(r)}\left(x \mid a_{1}, \ldots, a_{r} ; \lambda_{1}, \ldots, \lambda_{r}\right)\right\rangle \\
=\left\langle\int_{0}^{t}\left(\mathrm{Li}_{k}\left(1-e^{-s}\right)\right)^{\prime} d s \mid H_{n+1}^{(r)}\left(x \mid a_{1}, \ldots, a_{r} ; \lambda_{1}, \ldots, \lambda_{r}\right)\right\rangle \\
=\left\langle\int_{0}^{t} e^{-s} \frac{\mathrm{Li}_{k-1}\left(1-e^{-s}\right)}{1-e^{-s}} d s \mid H_{n+1}^{(r)}\left(x \mid a_{1}, \ldots, a_{r} ; \lambda_{1}, \ldots, \lambda_{r}\right)\right\rangle \\
=\left\langle\int_{0}^{t}\left(\sum_{j=0}^{\infty} \frac{(-s)^{j}}{j !}\right)\left(\sum_{m=0}^{\infty} \frac{B_{m}^{(k-1)}}{m !} s^{m}\right) d s \mid H_{n+1}^{(r)}\left(x \mid a_{1}, \ldots, a_{r} ; \lambda_{1}, \ldots, \lambda_{r}\right)\right\rangle \\
=\left\langle\sum_{l=0}^{\infty}\left(\sum_{m=0}^{l}(-1)^{l-m}\left(\begin{array}{c}
l \\
m
\end{array}\right) B_{m}^{(k-1)}\right) \frac{1}{l !} \int_{0}^{t} s^{l} d s \mid H_{n+1}^{(r)}\left(x \mid a_{1}, \ldots, a_{r} ; \lambda_{1}, \ldots, \lambda_{r}\right)\right\rangle \\
=\sum_{l=0}^{n} \sum_{m=0}^{l}(-1)^{l-m}\left(\begin{array}{c}
l \\
m
\end{array}\right) \frac{B_{m}^{(k-1)}}{(l+1) !}\left\langle t^{l+1} \mid H_{n+1}^{(r)}\left(x \mid a_{1}, \ldots, a_{r} ; \lambda_{1}, \ldots, \lambda_{r}\right)\right\rangle \\
=\sum_{l=0}^{n} \sum_{m=0}^{l}(-1)^{l-m}\left(\begin{array}{c}
l \\
m
\end{array}\right) \frac{B_{m}^{(k-1)}}{(l+1) !}(n+1)_{l+1} H_{n-l}^{(r)}\left(a_{1}, \ldots, a_{r} ; \lambda_{1}, \ldots, \lambda_{r}\right) \\
=\sum_{l=0}^{n} \sum_{m=0}^{l}(-1)^{l-m}\left(\begin{array}{c}
l \\
m
\end{array}\right)\left(\begin{array}{l}
n+1 \\
l+1
\end{array}\right) B_{m}^{(k-1)} H_{n-l}^{(r)}\left(a_{1}, \ldots, a_{r} ; \lambda_{1}, \ldots, \lambda_{r}\right) .
\end{aligned}
$$

Here, $H_{n-l}^{(r)}\left(a_{1}, \ldots, a_{r} ; \lambda_{1}, \ldots, \lambda_{r}\right)=H_{n-l}^{(r)}\left(0 \mid a_{1}, \ldots, a_{r} ; \lambda_{1}, \ldots, \lambda_{r}\right)$. Thus, we get (28). 


\subsection{Relations with the Stirling numbers of the second kind and the falling factorials}

\section{Theorem 6}

$$
\begin{aligned}
& T_{n}^{(r, k)}\left(x \mid a_{1}, \ldots, a_{r} ; \lambda_{1}, \ldots, \lambda_{r}\right) \\
& \quad=\sum_{m=0}^{n}\left(\sum_{l=m}^{n}\left(\begin{array}{l}
n \\
l
\end{array}\right) S_{2}(l, m) T_{n-l}^{(r, k)}\left(a_{1}, \ldots, a_{r} ; \lambda_{1}, \ldots, \lambda_{r}\right)\right)(x)_{m} .
\end{aligned}
$$

Proof For $(16)$ and $(x)_{n} \sim\left(1, e^{t}-1\right)$, assume that

$$
T_{n}^{(r, k)}\left(x \mid a_{1}, \ldots, a_{r} ; \lambda_{1}, \ldots, \lambda_{r}\right)=\sum_{m=0}^{n} C_{n, m}(x)_{m} .
$$

By (13), we have

$$
\begin{aligned}
C_{n, m} & =\frac{1}{m !}\left\langle\frac{1}{\prod_{j=1}^{r}\left(\frac{e^{a_{j} t}-\lambda_{j}}{1-\lambda_{j}}\right) \frac{1-e^{-t}}{\operatorname{Li}_{k}\left(1-e^{-t}\right)}}\left(e^{t}-1\right)^{m} \mid x^{n}\right\rangle \\
& =\frac{1}{m !}\left\langle\prod_{j=1}^{r}\left(\frac{1-\lambda_{j}}{e^{a_{j} t}-\lambda_{j}}\right) \frac{\mathrm{Li}_{k}\left(1-e^{-t}\right)}{1-e^{-t}} \mid\left(e^{t}-1\right)^{m} x^{n}\right\rangle \\
& =\frac{1}{m !}\left\langle\prod_{j=1}^{r}\left(\frac{1-\lambda_{j}}{e^{a_{j} t}-\lambda_{j}}\right) \frac{\mathrm{Li}_{k}\left(1-e^{-t}\right)}{1-e^{-t}} \mid m ! \sum_{l=m}^{n} S_{2}(l, m) \frac{t^{l}}{l !} x^{n}\right\rangle \\
& =\sum_{l=m}^{n}\left(\begin{array}{l}
n \\
l
\end{array}\right) S_{2}(l, m)\left\langle\prod_{j=1}^{r}\left(\frac{1-\lambda_{j}}{e^{a_{j} t}-\lambda_{j}}\right) \frac{\mathrm{Li}_{k}\left(1-e^{-t}\right)}{1-e^{-t}} \mid x^{n-l}\right\rangle \\
& =\sum_{l=m}^{n}\left(\begin{array}{l}
n \\
l
\end{array}\right) S_{2}(l, m) T_{n-l}^{(r, k)}\left(a_{1}, \ldots, a_{r} ; \lambda_{1}, \ldots, \lambda_{r}\right) .
\end{aligned}
$$

Thus, we get identity (29).

\subsection{Relations with the Stirling numbers of the second kind and the rising} factorials

Theorem 7

$$
\begin{aligned}
T_{n}^{(r, k)}\left(x \mid a_{1}, \ldots, a_{r} ; \lambda_{1}, \ldots, \lambda_{r}\right) \\
\quad=\sum_{m=0}^{n}\left(\sum_{l=m}^{n}\left(\begin{array}{l}
n \\
l
\end{array}\right) S_{2}(l, m) T_{n-l}^{(r, k)}\left(-m \mid a_{1}, \ldots, a_{r} ; \lambda_{1}, \ldots, \lambda_{r}\right)\right)(x)^{(m)} .
\end{aligned}
$$

Proof For (16) and $(x)^{(n)}=x(x+1) \cdots(x+n-1) \sim\left(1,1-e^{-t}\right)$, assume that $T_{n}^{(r, k)}\left(x \mid a_{1}, \ldots, a_{r}\right.$; $\left.\lambda_{1}, \ldots, \lambda_{r}\right)=\sum_{m=0}^{n} C_{n, m}(x)^{(m)}$. By (13), we have

$$
\begin{aligned}
C_{n, m} & =\frac{1}{m !}\left\langle\frac{1}{\prod_{j=1}^{r}\left(\frac{e^{a_{j} t}-\lambda_{j}}{1-\lambda_{j}}\right) \frac{1-e^{-t}}{\mathrm{Li}_{k}\left(1-e^{-t}\right)}}\left(1-e^{-t}\right)^{m} \mid x^{n}\right\rangle \\
& =\frac{1}{m !}\left\langle\prod_{j=1}^{r}\left(\frac{1-\lambda_{j}}{e^{a_{j} t}-\lambda_{j}}\right) \frac{\operatorname{Li}_{k}\left(1-e^{-t}\right)}{1-e^{-t}} e^{-m t} \mid\left(e^{t}-1\right)^{m} x^{n}\right\rangle
\end{aligned}
$$




$$
\begin{aligned}
& =\sum_{l=m}^{n}\left(\begin{array}{l}
n \\
l
\end{array}\right) S_{2}(l, m)\left\langle e^{-m t} \mid \prod_{j=1}^{r}\left(\frac{1-\lambda_{j}}{e^{a_{j} t}-\lambda_{j}}\right) \frac{\mathrm{Li}_{k}\left(1-e^{-t}\right)}{1-e^{-t}} x^{n-l}\right\rangle \\
& =\sum_{l=m}^{n}\left(\begin{array}{l}
n \\
l
\end{array}\right) S_{2}(l, m)\left\langle e^{-m t} \mid T_{n-l}^{(r, k)}\left(x \mid a_{1}, \ldots, a_{r} ; \lambda_{1}, \ldots, \lambda_{r}\right)\right\rangle \\
& =\sum_{l=m}^{n}\left(\begin{array}{l}
n \\
l
\end{array}\right) S_{2}(l, m) T_{n-l}^{(r, k)}\left(-m \mid a_{1}, \ldots, a_{r} ; \lambda_{1}, \ldots, \lambda_{r}\right) .
\end{aligned}
$$

Thus, we get identity (30).

\subsection{Relations with higher-order Frobenius-Euler polynomials}

Theorem 8

$$
\begin{aligned}
& T_{n}^{(r, k)}\left(x \mid a_{1}, \ldots, a_{r} ; \lambda_{1}, \ldots, \lambda_{r}\right) \\
& \quad=\sum_{m=0}^{n}\left(\frac{\left(\begin{array}{l}
n \\
m
\end{array}\right)}{(1-\lambda)^{s}} \sum_{l=0}^{s}\left(\begin{array}{l}
s \\
l
\end{array}\right)(-\lambda)^{s-l} T_{n-m}^{(r, k)}\left(l \mid a_{1}, \ldots, a_{r} ; \lambda_{1}, \ldots, \lambda_{r}\right)\right) H_{m}^{(s)}(x \mid \lambda) .
\end{aligned}
$$

Proof For (16) and

$$
H_{n}^{(s)}(x \mid \lambda) \sim\left(\left(\frac{e^{t}-\lambda}{1-\lambda}\right)^{s}, t\right),
$$

assume that $T_{n}^{(r, k)}\left(x \mid a_{1}, \ldots, a_{r} ; \lambda_{1}, \ldots, \lambda_{r}\right)=\sum_{m=0}^{n} C_{n, m} H_{m}^{(s)}(x \mid \lambda)$. By (13), we have

$$
\begin{aligned}
C_{n, m} & =\frac{1}{m !}\left\langle\left(\frac{e^{t}-\lambda}{1-\lambda}\right)^{s} \prod_{j=1}^{r}\left(\frac{1-\lambda_{j}}{e^{a_{j} t}-\lambda_{j}}\right) \frac{\mathrm{Li}_{k}\left(1-e^{-t}\right)}{1-e^{-t}} t^{m} \mid x^{n}\right\rangle \\
& =\frac{1}{m !(1-\lambda)^{s}} \sum_{l=0}^{s}\left(\begin{array}{l}
s \\
l
\end{array}\right)(-\lambda)^{s-l}\left\langle e^{l t} \prod_{j=1}^{r}\left(\frac{1-\lambda_{j}}{e^{a_{j} t}-\lambda_{j}}\right) \frac{\mathrm{Li}_{k}\left(1-e^{-t}\right)}{1-e^{-t}} \mid t^{m} x^{n}\right\rangle \\
& =\frac{\left(\begin{array}{c}
n \\
m
\end{array}\right)}{(1-\lambda)^{s}} \sum_{l=0}^{s}\left(\begin{array}{l}
s \\
l
\end{array}\right)(-\lambda)^{s-l}\left\langle e^{l t} \mid \prod_{j=1}^{r}\left(\frac{1-\lambda_{j}}{e^{a_{j} t}-\lambda_{j}}\right) \frac{\operatorname{Li}_{k}\left(1-e^{-t}\right)}{1-e^{-t}} x^{n-m}\right\rangle \\
& =\frac{\left(\begin{array}{l}
n \\
m
\end{array}\right)}{(1-\lambda)^{s}} \sum_{l=0}^{s}\left(\begin{array}{l}
s \\
l
\end{array}\right)(-\lambda)^{s-l} T_{n-m}^{(r, k)}\left(l \mid a_{1}, \ldots, a_{r} ; \lambda_{1}, \ldots, \lambda_{r}\right) .
\end{aligned}
$$

Thus, we get identity (31).

\subsection{Relations with higher-order Bernoulli polynomials}

Bernoulli polynomials $\mathfrak{B}_{n}^{(r)}(x)$ of order $r$ are defined by

$$
\left(\frac{t}{e^{t}-1}\right)^{r} e^{x t}=\sum_{n=0}^{\infty} \frac{\mathfrak{B}_{n}^{(r)}(x)}{n !} t^{n}
$$

(see, e.g., [4, Section 2.2]). 


\section{Theorem 9}

$$
\begin{aligned}
& T_{n}^{(r, k)}\left(x \mid a_{1}, \ldots, a_{r} ; \lambda_{1}, \ldots, \lambda_{r}\right) \\
& \quad=\sum_{m=0}^{n}\left(\begin{array}{c}
n \\
m
\end{array}\right)\left(\sum_{l=0}^{n-m} \frac{\left(\begin{array}{c}
n-m \\
l
\end{array}\right)}{\left(\begin{array}{c}
l+s \\
l
\end{array}\right)} S_{2}(l+s, s) T_{n-m-l}^{(r, k)}\left(a_{1}, \ldots, a_{r} ; \lambda_{1}, \ldots, \lambda_{r}\right)\right) \mathfrak{B}_{m}^{(s)}(x) .
\end{aligned}
$$

Proof For (16) and

$$
\mathfrak{B}_{n}^{(s)}(x) \sim\left(\left(\frac{e^{t}-1}{t}\right)^{s}, t\right)
$$

assume that $T_{n}^{(r, k)}\left(x \mid a_{1}, \ldots, a_{r} ; \lambda_{1}, \ldots, \lambda_{r}\right)=\sum_{m=0}^{n} C_{n, m} \mathfrak{B}_{m}^{(s)}(x)$. By (13), we have

$$
\begin{aligned}
C_{n, m} & =\frac{1}{m !}\left\langle\left(\frac{e^{t}-1}{t}\right)^{s} \prod_{j=1}^{r}\left(\frac{1-\lambda_{j}}{e^{a_{j} t}-\lambda_{j}}\right) \frac{\mathrm{Li}_{k}\left(1-e^{-t}\right)}{1-e^{-t}} t^{m} \mid x^{n}\right\rangle \\
& =\left(\begin{array}{c}
n \\
m
\end{array}\right)\left\langle\prod_{j=1}^{r}\left(\frac{1-\lambda_{j}}{e^{a_{j} t}-\lambda_{j}}\right) \frac{\mathrm{Li}_{k}\left(1-e^{-t}\right)}{1-e^{-t}} \mid\left(\frac{e^{t}-1}{t}\right)^{s} x^{n-m}\right\rangle \\
& =\left(\begin{array}{c}
n \\
m
\end{array}\right)\left\langle\prod_{j=1}^{r}\left(\frac{1-\lambda_{j}}{e^{a_{j} t}-\lambda_{j}}\right) \frac{\mathrm{Li}_{k}\left(1-e^{-t}\right)}{1-e^{-t}} \mid \sum_{l=0}^{n-m} \frac{s !}{(l+s) !} S_{2}(l+s, s) t^{l} x^{n-m}\right\rangle \\
& =\left(\begin{array}{c}
n \\
m
\end{array}\right) \sum_{l=0}^{n-m} \frac{s !}{(l+s) !} S_{2}(l+s, s)(n-m)_{l}\left\langle\prod_{j=1}^{r}\left(\frac{1-\lambda_{j}}{e^{a_{j} t}-\lambda_{j}}\right) \frac{\mathrm{Li}_{k}\left(1-e^{-t}\right)}{1-e^{-t}} \mid x^{n-m-l}\right\rangle \\
& =\left(\begin{array}{c}
n \\
m
\end{array}\right) \sum_{l=0}^{n-m} \frac{s !}{(l+s) !} S_{2}(l+s, s)(n-m)_{l} T_{n-m-l}^{(r, k)}\left(a_{1}, \ldots, a_{r} ; \lambda_{1}, \ldots, \lambda_{r}\right) \\
& =\left(\begin{array}{c}
n \\
m
\end{array}\right) \sum_{l=0}^{n-m} \frac{\left(\begin{array}{c}
l-m \\
\text { ( })
\end{array}\right)}{\left(\begin{array}{c}
l+s \\
l
\end{array}\right)} S_{2}(l+s, s) T_{n-m-l}^{(r, k)}\left(a_{1}, \ldots, a_{r} ; \lambda_{1}, \ldots, \lambda_{r}\right) .
\end{aligned}
$$

Thus, we get identity (32).

\section{Competing interests}

The authors declare that they have no competing interests.

\section{Authors' contributions}

All authors contributed equally to the manuscript and typed, read, and approved the final manuscript.

\section{Author details}

'Department of Mathematics, Sogang University, Seoul, 121-741, Republic of Korea. ${ }^{2}$ Department of Mathematics, Kwangwoon University, Seoul, 139-701, Republic of Korea. ${ }^{3}$ Department of Applied Mathematics, Pukyong National University, Pusan, 608-739, Republic of Korea. ${ }^{4}$ Graduate School of Science and Technology, Hirosaki University, Hirosaki, 036-8561, Japan.

\section{Acknowledgements}

The authors would like to thank the referees for their valuable comments and suggestions. This work was supported by the National Research Foundation of Korea (NRF) grant funded by the Korea government (MOE) (No.

2012R1A1A2003786). The fourth author was supported in part by the Grant-in-Aid for Scientific research (C) (No.

22540005), the Japan Society for the Promotion of Science. 


\section{References}

1. Bayad, A, Hamahata, Y: Polylogarithms and poly-Bernoulli polynomials. Kyushu J. Math. 65, 15-24 (2011)

2. Coppo, M-A, Candelpergher, B: The Arakawa-Kaneko zeta functions. Ramanujan J. 22, 153-162 (2010)

3. Kim, DS, Kim, T: Some identities of Frobenius-Euler polynomials arising from umbral calculus. Adv. Differ. Equ. 2012 Article ID 196 (2012)

4. Roman, S: The Umbral Calculus. Dover, New York (2005)

5. Kim, DS, Kim, T, Lee, S-H: Poly-Bernoulli polynomials arising from umbral calculus. Available at http://arxiv.org/pdf/1306.6697.pdf

10.1186/1687-1847-2014-92

Cite this article as: Kim et al.: Barnes' multiple Frobenius-Euler and poly-Bernoulli mixed-type polynomials. Advances in Difference Equations 2014, 2014:92

Submit your manuscript to a SpringerOpen ${ }^{\odot}$ journal and benefit from:

- Convenient online submission

- Rigorous peer review

Immediate publication on acceptance

- Open access: articles freely available online

- High visibility within the field

- Retaining the copyright to your article 